tional Science and Engineering Research Council (NSERC) and the Canadian Institute of Health Research (CIHR). Address correspondence to Dr. David W. Silversides, Faculty of Veterinary Medicine, University of Montreal, St-Hyacinthe, Québec, Canada.e-mail:silverdw@medvet.umontreal.ca

Received 21 August 2002; accepted 11 October 2002.

\author{
A. Boyer, M. Paquet, D. Rai- \\ wet, I. Daneau, W. Harrison ${ }^{1}$, \\ and D.W. Silversides \\ University of Montreal \\ St-Hyacinthe, Québec, Canada \\ ${ }^{1}$ Baylor College of Medicine \\ Houston, TX, USA
}

For reprints of this or any other article, contact Reprints@BioTechniques.com

\section{Deep Screening of Recombination Proficient Bacteriophage Libraries}

BioTechniques 34:36-40 (January 2003)

We are using bacteriophage $\lambda$ as an efficient vector for transferring genomic alterations to embryonic stem (ES) cells via gene targeting. We have developed a variety of phage-plasmid recombination techniques to direct mutations and modification cassettes to specific sites within phage targeting vectors (1,6-9). We have also made improvements on Seed's original method of recombination screening of bacteriophage libraries (5), which allows phage clones to be isolated "genetically" using recombination. In retro-recombination screening (8), phage targeting vectors from an ES cell targeting vector library in $\lambda$ thymidine kinase (TK) are purified on a restrictive host, following the homologous integration of a supF-homology-bearing plasmid, which suppresses $\mathrm{A}^{\mathrm{am}}, \mathrm{B}^{\mathrm{am}}$, and $\mathrm{S}^{\mathrm{am}}$ mutations in suitable phage $\lambda$ genes essential for phage growth. Because the region of homology is duplicated upon integration, and can therefore revert under relaxed conditions (i.e., within a $s u p F$ host), we incorporated the gam gene into the recombination plasmid to select for phages that have reverted to their original configuration (8). Retro-recombina- tion screening has several advantages over conventional phage library screening techniques. Specific targeting vector phages can be isolated without plaque hybridization and purified in 2-3 days to serve as templates for the rapid completion of targeting vector construction via recombination. Libraries are screened in culture tubes, meaning that multiple library screens (i.e., targeting vector homology region isolation) can be performed at one time and there is no shortage of the number of phages that can be screened simultaneously to ensure the isolation of distinct clones from the locus of interest. This latter point is particularly important in the case of our $\lambda \mathrm{TK}$ library because the insert size is approximately $12 \mathrm{~kb}$ and as many as $2 \times$ $10^{6}$ phages need to be screened at one time to ensure sufficient representation of all genes within the library.

One drawback of recombination screening approaches is that most libraries, including our $\lambda \mathrm{TK}$ library, are constructed with commercial packaging extracts that are not irradiated when prepared. As a result, a small yet significant number of non-amber phages can appear in a library of amber mutationcontaining phages, which arise from recombination between the two mutant strains used to generate head and tail extracts. While the incidence of such phages is rare (i.e., $10^{-4}$ ), it presents a unique challenge in screening amplified libraries by recombination, as

Table 1. Recombination Efficiencies

\begin{tabular}{|cccccc|}
\hline Samples & $\begin{array}{c}\text { Homology } \\
\text { (bp) }\end{array}$ & $\begin{array}{c}\text { Recombinant } \\
\text { (pfu/mL) }\end{array}$ & $\begin{array}{c}\text { Non-amber } \\
\text { (pfu/mL) }\end{array}$ & $\begin{array}{c}\text { Total } \\
(\mathbf{p f u} / \mathbf{m L})\end{array}$ & Frequency \\
\hline 1B2 & 249 & 90 & 188 & $8.0 \times 10^{9}$ & $1.1 \times 10^{-6}$ \\
3B6 & 515 & 60 & 246 & $1.0 \times 10^{9}$ & $6.0 \times 10^{-6}$ \\
6G2 & 452 & 20 & 223 & $1.0 \times 10^{9}$ & $2.0 \times 10^{-6}$ \\
7F5 & 570 & 30 & 157 & $1.3 \times 10^{8}$ & $2.3 \times 10^{-5}$ \\
12F2 & 433 & 10 & 145 & $1.0 \times 10^{8}$ & $1.0 \times 10^{-5}$ \\
1G7* & 337 & 100 & 460000 & $1.2 \times 10^{13}$ & $8.3 \times 10^{-10}$ \\
Following recombination screens, lysates were titered for blue (recombinant), \\
white (non-amber), and total pfus. The frequency of recombination is presented \\
as the number of blue plaques/total titer. ${ }^{*}$ 1G7 was precipitated from a 400-mL \\
culture using PEG and resuspended in a final volume of 0.4 mL. For 1G7, recom- \\
binant plaques were determined using DK21. For all others, recombinant plaques \\
were determined using LG75. Non-amber phages were identified as white \\
plaques on LG75. Total titers were determined using LE392. \\
\hline
\end{tabular}


these non-amber contaminant phages are also amplified in the liquid screens and confound high-density phage plating by appearing as positives when plated on a $\sup F^{0}$ host. This problem has been ameliorated somewhat by the use of LG75, a sup ${ }^{0}$ indicator host, which has an amber mutation in the lacZ gene (4). Using LG75, recombi- nant supF-bearing phages are identified as blue plaques among a number of non-blue contaminant phages.

Another problem that can arise in any library screen is that clones can become underrepresented whenever a library is amplified. This problem is accentuated in screening by recombination because an amplification step occurs

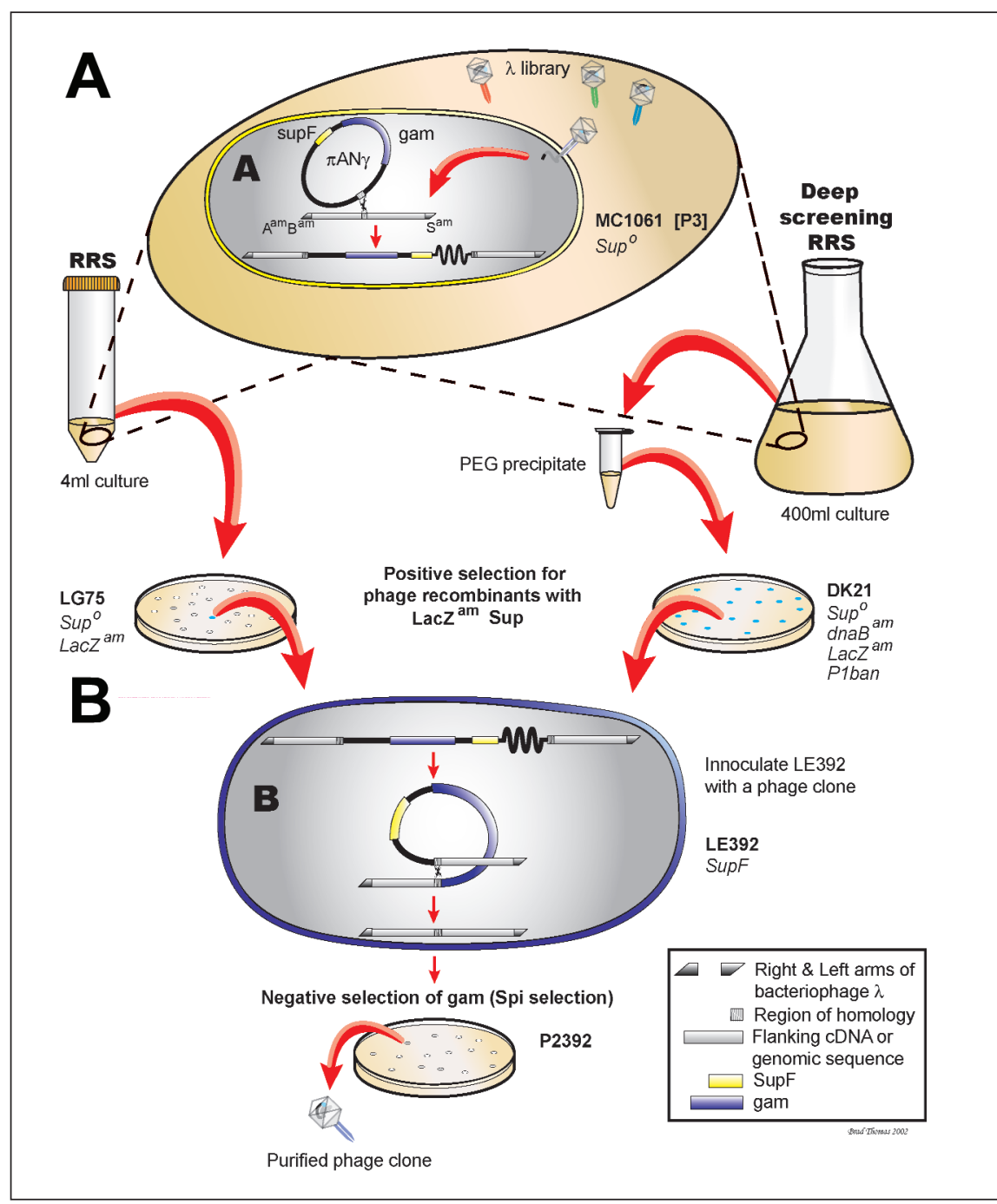

Figure 1. Flow diagram of the retro-recombination screening (RRS) and deep screening RRS procedures. (A) An aliquot of the $\lambda \mathrm{TK}$ targeting vector library is used to infect the rec ${ }^{+}$recombination plasmid host MC1061[p3]. Single crossover recombination between the probe homology and the corresponding homologous section in the cloned genomic region of interest (light blue) results in the integration of the probe plasmid into the phage. This phage-plasmid co-integrate contains a duplicated region of homology, as well as the supF and gam genes required for selection. Only single (or more rarely, odd number) crossover recombinants are capable of growth and blue plaque formation on a $\sup ^{0}$ host. In retro-recombination screening (RRS; left flowchart), an aliquot of a small liquid culture is plated onto the host LG75 ( $\mathrm{rec}^{+}$; sup ${ }^{0}$; lacZ $Z^{\mathrm{am}}$ ). Here a small percentage of non-amber (white) plaques are seen because of the use of a non-irradiated packaging extract during the preparation of the library. In deep screening RRS (right flowchart), a larger initial culture is used and concentrated using PEG precipitation. Here non-amber plaques are eliminated on DK21 (sup ${ }^{0}$; lacZ $Z^{\mathrm{am}} ;$ dnaB ${ }^{\mathrm{am}} ; \mathrm{P} 1$ ban). (B) Condensation of the recombinant phage in LE392 $\left(\mathrm{rec}^{+}\right.$; supE; supF) allows for a second recombination event to occur between the duplicate regions of homology. Such recombinants lose the gam gene and are capable of growth on P2392 (P2 lysogen of LE392). when the library is screened in liquid culture. Hence, clones that are 100-fold less abundant in the original library might become 10000 -fold less abundant during the screening step.

Using retro-recombination screening, we recently attempted the parallel isolation of a variety of $\lambda \mathrm{TK}$ genomic clones representing six expressed sequence tags we previously identified following the neural differentiation of ES cells (2). During this screen, however, one of these clones, $1 \mathrm{G} 7$, was refractory to our normal isolation procedure. This situation can occur either because the homology region in the probe plasmid is of insufficient length for efficient isolation of a recombinant or the gene is less abundant in the library. Increasing the sensitivity of the recombination screening may alleviate each of these potential problems. Accordingly, we have developed a method of deep screening, which increases the sensitivity of recombination screening as much as 100000 -fold (Table 1). Using this approach, we were able to isolate multiple recombinant clones for the expressed sequence tag 1G7 successfully.

Our deep screening method takes advantage of the fact that a much larger culture volume can be used to screen for underrepresented recombination events. Moreover, concentrating phage lysates using PEG precipitation can further enhance the presentation of those underrepresented recombination events.

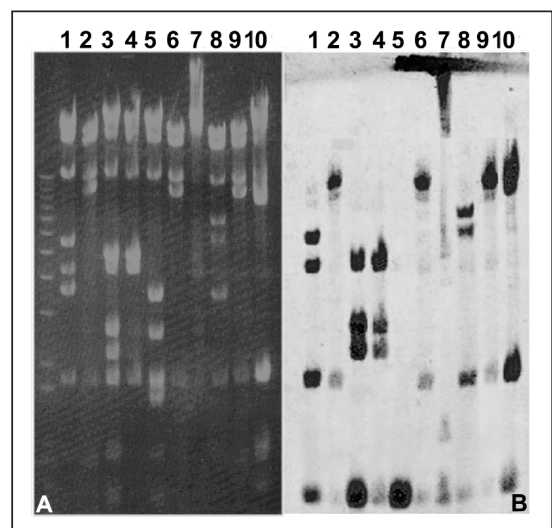

Figure 2. Analysis of $\mathbf{1 G 7}$ clones isolated by deep screening. DNA preparations from 10 clones were chosen at random, digested with Sall and EcoRI, and subjected to Southern blot analysis using a $1 \mathrm{G} 7$ probe cDNA probe lying outside the homology region used in the screen. All of the selected clones were positive for the locus based on multiple hybridizing fragments. 
While normally we will pass $3 \times 10^{7}$ pfu in a 4-mL culture, 100-fold more phage may be screened in a $400-\mathrm{mL}$ culture volume to help in the identification of underrepresented clones within the library. Using PEG precipitation, we were able to further concentrate this volume 1000 -fold. After clarifying the resuspended phage pellet using chloroform extraction, the 100000 -fold concentrated lysate can be passaged over the indicator host for identification of recombinants. Here, however, we ran into significant difficulties due to the presence of non-amber-bearing phages in the original library. While in normal screens these phages are easily detected on the indicator host LG75, in phage extracts that have been concentrated up to 100000 -fold, the significant number of contaminating plaques that are observed (Table 1) generates confluent lysis on LG75 indicator plates, preventing the detection of blue "recombinant" plaques. To circumvent this problem, it was necessary to use an indicator host, which was able to eliminate these nonamber bacteriophage while retaining the ability to detect recombinants. Here we were able to use the indicator host DK21, which has amber mutations in both the $l a c Z$ and $d n a B$ genes (3). Because $d n a B$ is necessary for bacteriophage replication, yet is dispensable for $E$. coli chromosomal replication when complemented by the P1ban gene in DK21, only recombinant bacteriophages bearing supF plasmid integrations are able to grow. Accordingly, on DK21, all non-recombinant phages, including the non-amber contaminants, are eliminated. Using the large-scale screen, we were able to detect multiple recombinant plaques out of approximately $10^{13}$ phages that were screened (Table 1). Because bacteriophages carrying supF grow relatively slowly on DK 21 , infection is performed on $\lambda$ tryptone minimal medium to slow bacterial growth and allow the plaques to become large enough to easily detect lacZ expression (3). Following the isolation of blue plaques on DK21, individual recombinants are sequentially passaged over the supF hosts LE392 and P2392, as we routinely do (Figure 1), to allow excision of the plasmid integration. Upon isolation of these rare recombi- nants from the library, DNA was prepared from these 10 recombinants and Southern blotted using a 1G7 probe to confirm that they were associated with the $1 \mathrm{G} 7$ locus (Figure 2).

While deep screening may be a useful technique for isolating underrepresented genes within libraries, it also will have utility in other applications involving phage-plasmid recombination. We have recently developed novel transplacement technology that allows both point mutations and gene insertions/deletions to be engineered into specific phages via integrative and excisive recombination $(1,7,9$; Woltjen et al., in preparation). Because this technology is identical to the recombination cycles that occur in retro-recombination screening, we have demonstrated that retro-recombination screening and transplacement can be combined to isolate phages genetically and deposit mutations at the same time. While currently we are using considerable homology lengths (i.e., 300-500 bp) to facilitate these recombination events, the increased sensitivity of deep screening will allow the isolation of rare recombination events that will occur as a result of using smaller regions of homology. At the same time, the increased sensitivity of deep screening may also increase the likelihood of identifying false-positive recombination events. Accordingly, care will need to be taken to ensure that recombinants that are isolated are true.

\section{REFERENCES}

1.Aoyama, C., K. Woltjen, F.C. Mansergh, K. Ishidate, and D.E. Rancourt. Bacteriophage gene targeting vectors generated by transplacement. BioTechniques (In Press.)

2.Bain, G., F.C. Mansergh, M.A. Wride, J.E. Hance, A. Isogawa, S.L. Rancourt, W.J. Ray, Y. Yoshimura, et al. 2000. ES cell neural differentiation reveals a substantial number of novel ESTs. Funct. Integr. Genomics 1:127-139.

3.Kurnit, D.M. and B. Seed. 1990. Improved genetic selection for screening bacteriophage libraries by homologous recombination in vivo. Proc. Natl. Acad. Sci. USA 87:31663169.

4.Lutz, C.T., W.C. Hollifield, B. Seed, J.M. Davie, and H.V. Huang. 1987. Syrinx 2A: an improved lambda phage vector designed for screening DNA libraries by recombination in vivo. Proc. Natl. Acad. Sci. USA. 84:43794383.
5.Seed, B. 1983. Purification of genomic sequences from bacteriophage libraries by recombination and selection in vivo. Nucleic Acids Res 11:2427-2445

6.Tsuzuki, T. and D.E. Rancourt. 1998. Embryonic stem cell gene targeting using bacteriophage lambda vectors generated by phageplasmid recombination. Nucleic Acids Res. 26:988-993

7.Unger, M.W.T., S.Y. Liu, and D.E. Rancourt. 1999. Transplacement mutagenesis: a novel in situ mutagenesis system using phageplasmid recombination. Nucleic Acids Res 27:1480-1484.

8.Woltjen, K., G. Bain, and D.E. Rancourt. 2000. Retro-recombination screening of a mouse embryonic stem cell genomic library. Nucleic Acids Res. 28e14:1-7.

9.Woltjen, K., M.W. Unger, and D.E. Rancourt. 2002. Transplacement mutagenesis. A recombination-based in situ mutagenesis protocol. Methods Mol. Biol. 182:189-207.

We thank David Kurnit for the indicator strain DK21. B.T. is supported by a studentship from The Network Centres of Excellence in Stem Cell Biology. K.W. was funded by studentships from the Alberta Heritage Foundation for Medical Research and the National Science and Engineering Council of Canada. D.E.R. is a scholar of the Alberta Heritage Foundation for Medical Research. Address correspondence to Dr. Derrick E. Rancourt 374 HMRB, 3330 Hospital Dr. N.W., Calgary, AB, T2N4N1, Canada.e-mail: rancourt@ucalgary.ca

Received 25 July 2002; accepted 22 October 2002.

\section{Brad Thomas, Knut Woltjen, and Derrick E. Rancourt University of Calgary Calgary, Alberta, Canada}

For reprints of this or any other article, contact Reprints@BioTechniques.com 